\title{
Association between necropsy evidence of disseminated intravascular coagulation and coagulation variables before death in patients in intensive care units
}

\author{
J T WILDE, K M ROBERTS, * M GREAVES, F E PRESTON \\ From the University Departments of Haematology and ${ }^{*}$ Histopathology, Royal Hallamshire Hospital, Sheffield
}

SUMMARY The necropsy findings in 21 patients on an intensive care unit, on whom coagulation studies had been performed immediately before death, were assessed. Eleven of the patients were retrospectively studied and 10 were reviewed consecutively in a prospective study. Fifteen patients (eight retrospective and seven prospective) had evidence of disseminated intravascular coagulation. Microthrombi were most often found in the lungs and kidneys. The most common abnormal coagulation tests in patients with necropsy evidence of disseminated intravascular coagulation were raised serum concentrations of fibrinogen and fibrin degradation products, prolonged prothrombin time, and reduced platelet counts. Reduced fibrinogen concentrations and a prolonged thrombin time were the least commonly observed abnormalities. There was no difference in either the prevalence or magnitude of abnormality of any particular coagulation variable test result between those patients with evidence of disseminated intravascular coagulation at necropsy and those without.

Abnormalities of coagulation often occur in patients on intensive care units. The causes of these abnormalities are numerous and often multifactorial. A common cause is disseminated intravascular coagulation.

Although many reports have described the necropsy findings in patients diagnosed as having disseminated intravascular coagulation on clinical and laboratory grounds during their terminal illness,${ }^{1-7}$ few mention the incidence of histologically confirmed disseminated intravascular coagulation at necropsy in such patients. We are not aware of any study which has analysed coagulation data before death to determine whether any differences exist between such patients who do and do not have evidence of disseminated intravascular coagulation at necropsy.

We performed a detailed histological analysis of necropsy material obtained from patients on intensive care units in whom coagulation data were available immediately before death. We looked in particular for evidence of disseminated intravascular coagulation. The findings in patients with and without evidence of disseminated intravascular coagulation at necropsy were compared.

Accepted for publication 5 August 1987

\section{Material and methods}

Twenty one patients ( 11 men, 10 women) who died on the intensive care unit of this hospital were included in the study. The mean age was 60 years (range 19-76). The study population was derived by combining patients from two separate studies. To satisfy the main aim of the study - to relate coagulation findings at the time of death to pathological appearances at necropsy-it was decided to analyse only subjects in whom coagulation tests had been performed within 24 hours of death. In an initial retrospective study necropsy material was analysed from 11 randomly selected patients who had died between October 1981 and April 1983. During this period a total of 136 patients died on the unit, and 87 post mortem examinations were performed. In a further prospective study necropsy material from 10 consecutive patients, in whom coagulation tests had been performed before death, was reviewed. Deaths had occurred between March and June, 1985. During this period there were 25 deaths on the unit and 15 post mortem examinations.

In each case tissue samples were examined from the following organs: heart, lung, kidney, liver, spleen, brain, adrenal, pancreas, thyroid. Tissue samples were embedded in paraffin wax. Sections were cut at $4 \mu \mathrm{m}$ 
and stained with haematoxylin and eosin, phosphotungstic acid haematoxylin, and Martius scarlet blue for the identification of fibrin microthrombi. Disseminated intravascular coagulation was diagnosed at necropsy by the finding of at least one microthrombus in two or more organs and absence of any local disease which may have been associated with microthrombus formation.

The results of the following laboratory tests taken before death were reviewed: prothrombin time (normal 11-15 seconds); activated partial thromboplastin time (normal 32-45 seconds); thrombin time (normal $\leqslant 14$ seconds); fibrinogen, measured by the Clauss method $^{8}$ (normal 1.6-3.9 g/1); Thrombo-Wellcotest serum fibrinogen/fibrin degradation products (normal $<8 \mathrm{ug} / \mathrm{ml}$ ); and platelets, measured on the Technicon Haemalog 8 autoanalyser (normal 150-400 $\times 10^{9} / 1$ ).

\section{Results}

Of the 21 patients included in this study, 15 had evidence of disseminated intravascular coagulation.
These comprised eight of the 11 from the retrospective study and seven of the 10 from the prospective study (nine men and six women). The underlying diagnoses in these patients were as follows: septicaemia $(n=5)$; ruptured aortic aneurysm ( $n=5)$; post-vascular surgery $(n=2)$; myocardial infarction $(n=2)$; multiple trauma following road traffic accident $(n=1)$. The percentage frequency of the finding of

Table 1 Prevalence (\%) of fibrin microthrombi in individual organs. (No of patients in whom particular organ $(s)$ examined in parentheses)

\begin{tabular}{lc}
\hline Site & $\begin{array}{l}\text { Percentage prevalence of fibrin } \\
\text { microthrombi }\end{array}$ \\
\hline Lung $(\mathrm{n}=15)$ & 87 \\
Kidney $(\mathrm{n}=14)$ & 100 \\
Adrenal $(\mathrm{n}=11)$ & 55 \\
Central nervous system $(\mathrm{n}=8)$ & 75 \\
Pancreas $(\mathrm{n}=10)$ & 60 \\
Thyroid $(\mathrm{n}=7)$ & 29 \\
Spleen $(\mathrm{n}=7)$ & 43 \\
Myocardium $(\mathrm{n}=15)$ & 47 \\
Liver $(\mathrm{n}=14)$ & 21 \\
\hline
\end{tabular}

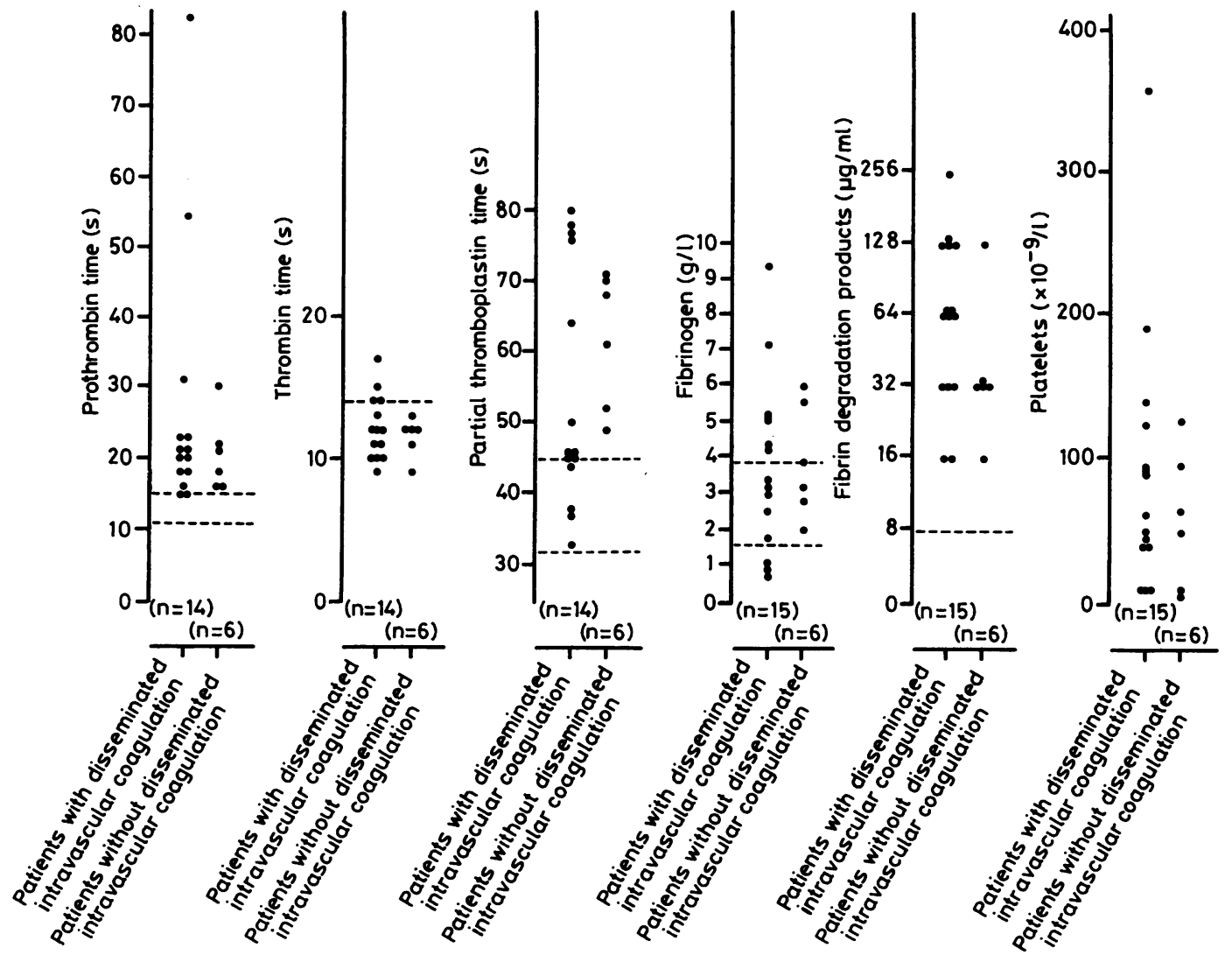

Figure Comparison of coagulation variable test results between patients before and after death with and without evidence of disseminated intravascular coagulation at necropsy. 
Table 2 Prevalence (\%) of haemorrhage in individual organs.

\begin{tabular}{ll}
\hline Site & $\begin{array}{l}\text { Percentage prevalence of } \\
\text { haemorrhage }\end{array}$ \\
\hline Lung $(\mathrm{n}=15)$ & 93 \\
Kidney $(\mathrm{n}=14)$ & 86 \\
Adrenal $(\mathrm{n}=11)$ & 73 \\
Central nervous system $(\mathrm{n}=11)$ & 50 \\
Pancreas $(\mathrm{n}=10)$ & 50 \\
Thyroid $(\mathrm{n}=7)$ & 43 \\
Spleen $(\mathrm{n}=7)$ & 29 \\
Myocardium $(\mathrm{n}=15)$ & 27 \\
Liver $(\mathrm{n}=14)$ & 7
\end{tabular}

fibrin microthrombi in individual organs is shown in table 1 . The lungs and the kidneys were the most commonly affected organs. The prevalence of haemorrhage in individual organs is shown in table 2. Again, the lungs and the kidneys were the organs most often affected.

One patient had abnormalities of all six coagulation variables measured before death, one had five abnormal tests, six had four abnormal tests, five had three abnormal tests, and two had two abnormal tests. One patient had been receiving warfarin immediately before death so the prothrombin time result was not included in the analysis. The blood sample from another patient was contaminated with heparin so the results of the thrombin time and activated partial thromboplastin time were excluded.

The causes of death in the six patients with no evidence of disseminated intravascular coagulation at necropsy were as follows: septicaemia $(n=3)$; acute pancreatitis $(n=2)$; ruptured aortic aneurysm $(n=$ 1). Five patients had four abnormal coagulation tests before death and one had three abnormal tests.

The figure compares the results of the individual coagulation variables of both groups.

There was no apparent difference in the length of time from the onset of the coagulation abnormalities until death between the two groups. In the group with disseminated intravascular coagulation the mean time interval was three days with a range of 10 days to less than 24 hours, and in the other group the mean time interval was two days with a range of five days to less than 24 hours. There was no major difference in the time interval between death and post mortem examination; the mean was 34 hours in the group with disseminated intravascular coagulation (range three89 hours) and 44 hours in the group without disseminated intravascular coagulation (range 12-96 hours).

\section{Discussion}

The pathological diagnosis of disseminated intravascular coagulation is based primarily on the finding of fibrin microthrombi in arterioles, capillaries, and venules in the absence of any local disease which may have accounted for their deposition. ${ }^{1569}$

There is no general agreement as to how many fibrin microthrombi need to be observed before disseminated intravascular coagulation can be diagnosed at necropsy. A recent study by Tanaka, ${ }^{5}$ who assessed the findings of consecutive necropsies for evidence of disseminated intravascular coagulation, required the presence of microthrombi in at least three separate organs for a definite diagnosis to be made. Robboy $e t$ al' suggest that the finding of even one fibrin microthrombus at necropsy is presumptive evidence for the existence of disseminated intravascular coagulation. In our study we decided to take the finding of fibrin microthrombi in a minimum of two separate organs as indicative of the presence of disseminated intravascular coagulation; 14 of the 15 patients who were found to have microthrombi had at least three of the organs analysed, affected.

Macroscopically, the pathological lesions that might be expected at necropsy in cases of disseminated intravascular coagulation are not always obvious. Cursory microscopic inspection of tissues may also fail to identify fibrin thrombi in blood vessels. ${ }^{47}$ Detailed microscopic assessment must therefore be made by ensuring adequate numbers of blocks are taken for histological analysis from numerous organs. This is important because the organs affected by disseminated intravascular coagulation vary from case to case, and there can be considerable histological variation within the same organ. It may be that there are pronounced variations in the fibrinolytic activity of small blood vessels from organ to organ within the same patient and from patient to patient. ${ }^{410}$

In our study microthrombi were most often found in the lungs and kidneys. This is in keeping with the findings of other studies reporting necropsy changes associated with disseminated intravascular coagulation. ${ }^{25}$

McKay" and Davies-Jones et al $^{12}$ postulated that during disseminated intravascular coagulation microthrombi often form in the systemic circulation and are sieved off by the microvasculature of the organs through which they pass. It is therefore not surprising that organs which receive the highest percentage of cardiac output per unit size, such as the lungs and kidneys, are the most common sites in which microthrombi are found.

No single laboratory test, nor any particular combination of tests, can be used to make a definite diagnosis of disseminated intravascular coagulation. ${ }^{13}$ Abnormalities of the routinely performed coagulation tests can only lend support to a working diagnosis of disseminated intravascular coagulation in a patient with an associated aetiological condition who has evidence of generalised organ dysfunction or a bleed- 
ing tendency, or both. Because disseminated intravascular coagulation is such a dynamic process, results of coagulation tests may continually change. A single set of results should therefore be interpreted with caution. Sequential testing is much more informative as it establishes the pattern of change, be it improvement or deterioration with time. ${ }^{14}$.

Reviewing the coagulation data obtained not more than 24 hours before death in the 15 patients with necropsy evidence of disseminated intravascular coagulation, the most common abnormal tests were raised serum fibrinogen/fibrin degradation products $(100 \%)$, prolonged prothrombin time $(86 \%)$, and reduced platelets $(87 \%)$. A prolonged activated partial thromboplastin time was relatively insensitive $(50 \%)$. The most insensitive tests were a reduced fibrinogen $(20 \%)$ and a prolonged thrombin time $(14 \%)$.

Many studies have reviewed the prevalence of coagulation test abnormalities in disseminated intravascular coagulation. ${ }^{15-21}$ It is very difficult to make comparative analyses between the results obtained in these studies due to the heterogeneity of the criteria laid down for the diagnosis of disseminated intravascular coagulation. Another problem arises in interpretation because in these studies results from patients with disseminated intravascular coagulation of wide ranging severity have been pooled together.

The results of our study are in general agreement with those of other studies: the most sensitive of the routinely performed tests used in the investigation of disseminated intravascular coagulation seems to be a prolongation of prothrombin time, an increase in fibrinogen/fibrin degradation products, and a reduction in platelets. A prolonged thrombin time, though, seems to be a more common accompaniment of disseminated intravascular coagulation in other studies. A possible explanation of this is that in most reports the patients were investigated on account of a haemorrhagic diathesis; this was not a major feature in our patients.

The findings of our study also agree with those of other studies in that the fibrinogen concentration is an insensitive indicator of disseminated intravascular coagulation. Normal fibrinogen concentrations may be found in disseminated intravascular coagulation for two main reasons. Firstly, fibrinogen concentrations may be raised as a consequence of malignancy or pregnancy before the onset of disseminated intravascular coagulation. Although high consumption of fibrinogen may occur because of disseminated intravascular coagulation, concentrations may not fall below the normal range.$^{10}$ Secondly, in milder forms of disseminated intravascular coagulation increased liver synthesis of fibrinogen may balance consumption and, paradoxically, increased concentrations may even be observed..$^{10}$
Twelve of our patients with necropsy changes of disseminated intravascular coagulation had serial coagulation results. Of these, four showed a reduction in fibrinogen concentrations from the time of initial testing to those obtained before death. The eight other patients all showed an increase in fibrinogen concentrations and, interestingly, in six of these, concentrations were above the normal range.

There did not appear to be any differences either in the prevalence or magnitude of abnormality of any individual coagulation variable between those patients with and without evidence of disseminated intravascular coagulation at necropsy. In fact, all six patients without microthrombi deposition fulfilled the simple diagnostic criteria for disseminated intravascular coagulation as used by Minna ${ }^{2}$ - that is the presence of at least three of the four following findings-prolonged prothrombin time; reduced fibrinogen; reduced platelet count; raised conventions of fibrinogen/fibrin degradation products. Furthermore, using the more complicated diagnostic scoring system suggested by Kobayashi, ${ }^{21}$ three had definite disseminated intravascular coagulation and three probable. These findings would suggest that the coagulation test abnormalities usually taken to indicate the presence of disseminated intravascular coagulation are not specific for this condition. It may be, though, that disseminated intravascular coagulation was present in this group of patients and there are several reasons why confirmatory evidence of this was not found at necropsy.

Firstly, although necropsy material was thoroughly examined, deposited microthrombi may have been missed. Secondly, fibrinolysis of deposited microthrombi after death may have occurred in these patients. Another possibility is that, although there was a definite intravascular activation of coagulation in these patients with formation of circulating soluble fibrin complexes, these were not deposited as microthrombi. It has previously been reported that microthrombi deposition is not always found at post mortem examination in cases in which disseminated intravascular coagulation has already been diagnosed on clinical and laboratory grounds. Tanaka and Imamura $^{5}$ reviewed 109 such cases and found no evidence of microthrombi deposition in 67 of them.

We conclude from our study that disseminated intravascular coagulation seems to be a common occurrence in patients dying on the intensive care unit, although, because of our study design, we are unable to draw any conclusion as to its actual incidence. Abnormalities of the routinely used coagulation variables do not seem to be useful in predicting those cases in whom histological evidence of disseminated intravascular coagulation will be found at necropsy. Supplementary tests which specifically detect lytic 
products of deposited crosslinked fibrin, such as Ddimer estimation, may in future prove to be more accurate in determining those patients who have evidence of disseminated intravascular coagulation at necropsy.

\section{References}

1 Robboy SJ, Colman RW, Minna JD. Pathology of disseminated intravascular coagulation (D.I.C.)-analysis of 26 cases. Hum Pathol 1972;3:327-43.

2 Minna JD, Robboy SJ, Colman RW. Disseminated intravascular disease in man. Springfield, Illinois: Charles C Thomas, 1974.

3 Preston FE, Malia RG, Sworn MJ, Timperley WR, Blackburn EK. Disseminated intravascular coagulation as a consequence of cerebral damage. J Neurol Neurosurg Psychiatry 1974; 37:241-8.

4 Timperley WR. Disseminated intravascular coagulation in forensic pathology. Med Sci Law 1978;18:108-16.

5 Tanaka K, Imamura T. Incidence and clinicopathological significance of D.I.C. in autopsy cases. Bibl Haematol 1983;49: 73-93.

6 Kojima M, Shimamura K, Mori N, Oka K, Nakazawa M. A histological study on microthrombi in autopsy cases of D.I.C. Bibl Haematol 1983;49:95-106.

7 Kaufman HH, Hui K-S, Mattson JC, et al. Clinicopathological correlation of D.I.C. in patients with head injury. Neurosurgery 1984;15:34-42.

8 Dacie JV, Lewis SM. Practical haematology. 6th Ed. Edinburgh: Churchill Livingstone, 1984:229.

9 Kim H-S, Suzuki M, Lie JT, et al. Clinically unsuspected D.I.C.an autopsy survey. Am J Clin Pathol 1976;66:31-9.

10 Preston FE. Disseminated intravascular coagulation. Br J Hosp Med 1982;28:129-37.
11 McKay DG. Progress in disseminated intravascular coagulation. Calif Med 1969;111:186-99.

12 Davies-Jones GAB, Preston FE, Timperley WR. Neurological complications in clinical haematology. Oxford: Blackwell Scientific, 1980:193.

13 Hamilton PJ, Stalker AL, Douglas AS. Disseminated intravascular coagulation: a review. J Clin Pathol 1978;31:609-19.

14 Sharp AA. Diagnosis and management of disseminated intravascular coagulation. Br Med Bull 1977;33:265-73.

15 Spero JA, Lewis JH, Hasiba U. Disseminated intravascular coagulation-findings in 346 patients. Thromb Haemost 1980; 43:28-33.

16 Colman RW, Robboy SJ, Minna JD. disseminated intravascular coagulation-an approach. Am J Med 1972;52:679-99.

17 Cooper HA, Bowie EJW, Owen CA. Evaluation of patients with increased fibrinolytic split products (FSP) in their serum. Mayo Clin Proc 1974;49:654-7.

18 Al-Mondhiry H. Disseminated intravascular coagulationexperience in a major cancer centre. Thromb Diath Haemorrhagica 1975;34:181-93.

19 Lewis JH, Spero JA, Hasiba U. Coagulopathies. Disease a Month 1977;23:43-9.

20 Seigal T, Seligsoh U, Aghai E. Clinical and Laboratory aspects of D.I.C.-A study of 118 cases. Thromb Haemost 1978;39: 122-34.

21 Kobayashi N, Maekawa T, Takada M, Tanaka H, Gonmori H. Criteria for diagnosis of D.I.C. based on the analysis of clinical and laboratory findings in 345 D.I.C. patients collected by the research committee on D.I.C. in Japan. Bibl Haematol 1983;49:265-75.

Requests for reprints to: $\operatorname{Dr} J \mathrm{~T}$ Wilde, Lecturer in Haematology, Royal Hallamshire Hospital, Glossop Road, Sheffield S10 2JF, England. 